\title{
Aneurismas de la aorta torácica. Historia natural, diagnóstico y tratamiento
}

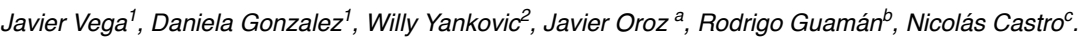 \\ 1. Residente Cirugía, Hospital San Juan de Dios, Santiago, Chile. \\ 2. Jefe Servicio Cirugía Vascular, Hospital San Juan de Dios, Santiago, Chile. \\ a. Interno, Facultad de Medicina, Universidad Andrés Bello, Viña del Mar, Chile. \\ b. Interno, Facultad de Medicina, Sede Occidente, Universidad de Chile, Santiago, Chile. \\ c. Alumno, Facultad de Medicina, Sede Occidente, Universidad de Chile, Santiago, Chile.
}

Los aneurismas corresponden a una dilatación arterial localizada que es producida por una debilidad de la pared; se clasifican en verdaderos y falsos. La incidencia es de aproximadamente 6 - 10 casos por 100.000 habitantes al año y los aneurismas de la aorta ascendente son los más frecuentes dentro del grupo de los aneurismas de la aorta torácica (AAT). Habitualmente se generan como resultado de una necrosis quística de la media que suele estar asociada a otras patologías. Los aneurismas suelen pesquisarse de manera incidental mediante exámenes imagenológicos solicitados por diferentes motivos. Su historia natural no está completamente clarificada, pero en términos generales los AAT tienen un crecimiento promedio de $0,1 \mathrm{~cm}$ por año. La angiografía por tomografía computada o por resonan- cia magnética, son los exámenes de elección para el diagnóstico. Para aquellos pacientes con aneurismas pequeños que no requieren cirugía, las medidas orientadas a la reducción del riesgo de ateroesclerosis son el pilar del tratamiento. Para los aneurismas de la aorta ascendente que cumplan con los requisitos, requieran de revascularización coronaria o reemplazo valvular, se recomienda el tratamiento quirúrgico. En relación a los aneurismas del cayado aórtico las opciones terapéuticas son tres: cirugía, terapia endovascular y terapia combinada. Para los aneurismas de la aorta descendente la terapia endovascular se perfila como la más eficiente. Los pacientes sin indicación de tratamiento quirúrgico o endovascular deben ser controlados de manera seriada con estudios imagenológicos.

\section{Correspondencia:}

Dr. Javier Vega S.

Huérfanos 3255, Santiago de Chile

drjaviervega@gmail.com 


\section{Thoracic aortic aneurysm. Natural history, diagnosis and management}

Aneurysms are defined as a localized dilatation caused by a weakness in the arterial wall. and may be classified as true or false aneurysm. The incidence is estimated to be 6-10 cases per 100,000 patient years. Aneurysms of the ascending aorta are the most common within the group of thoracic aortic aneurysms (TAA). These often result from cystic medial degeneration, which tends to be associated with other pathologic entities. Aneurysms are diagnosed most commonly on imaging studies performed for an unrelated indication. Although its natural history remains unclear, it is known that current average growth is about $0.1 \mathrm{~cm}$ per year. Computed tomography angiography (CTA) and magnetic resonance angiography (MRA) are the preferred imaging tests to detect aor- tic aneurysms. Measures aimed to reduce atherosclerosis risk are the main treatment objective for patients with smaller aneurysms that do not require surgery. When ascending aortic aneurysms meet the size criteria or coronary revascularization and aortic valvular replacement are necessary, surgical treatment it's recommended. For aortic arch aneurysms, there are three therapeutic alternatives: surgery, endovascular therapy and combined therapy. For descending aortic aneurysms, endovascular therapy is the preferred procedure. Patients with no indication for surgical or endovascular therapy, should be followed up with serial imaging studies.

Key words: thoracic aortic aneurysms; natural history; management. 


\section{Definición y clasificación}

Los aneurismas se definen como una dilatación arterial localizada, que es producida por una debilidad de la pared (congénita o adquirida). Esta dilatación debe ser mayor al $50 \%$ del diámetro normal del vaso ${ }^{1}$. El diámetro aórtico varía dependiendo del segmento estudiado, el género, la edad y la talla del paciente, que han permitido establecer rangos de normalidad (Tabla 1 ).

\begin{tabular}{|c|c|}
\hline \multicolumn{2}{|c|}{$\begin{array}{l}\text { Tabla 1. Diámetros normales de la aorta torácica } \\
\text { en adultos }(\mathrm{cm})^{2} \text {. }\end{array}$} \\
\hline Aorta ascendente (hombre, mujer) & 2,86 \\
\hline Arco aórtico (hombre) & 3,63 a 3,91 \\
\hline Arco aórtico (mujer) & 3,50 a 3,72 \\
\hline Aorta descendente proximal (hombre) & 2,39 a 2,98 \\
\hline Aorta descendete proximal (mujer) & 2,45 a 2,64 \\
\hline Aorta descendente distal (hombre) & 2,43 a 2,69 \\
\hline Aorta descendente distal (mujer) & 2,40 a 2,44 \\
\hline
\end{tabular}

Los aneurismas pueden ser clasificados en dos tipos: aneurisma verdadero (fusiforme o sacular) y aneurisma falso (pseudoaneurisma) ${ }^{1}$ (Figura 1).

Se habla de un aneurisma verdadero cuando están afectadas las tres paredes arteriales (íntima, media y adventicia). En el subtipo fusiforme hay una dilatación simétrica de toda la circunferencia, mientras que en el sacular hay una dilatación más localizada, semejante a una evaginación ${ }^{3}$.

El pseudoaneurisma se forma cuando existe una sección parcial de la pared de una arteria. La sangre que escapa del vaso genera un hematoma, el que posteriormente se organiza creando una pseudocápsula fibrosa ${ }^{4}$.

\section{Epidemiología}

La incidencia de los aneurismas de la aorta torácica (AAT) es de aproximadamente 6 - 10 casos por 100.000 habitantes al año ${ }^{5}$ y ha aumentado dada la mejora en los métodos de tamizaje y los avances en imagenología 6 . El peak se presenta entre la sexta y séptima década de la vida, siendo los hombres $2-4$ veces más afectados que las mujeres ${ }^{1}$. De todos los AAT, los que comprometen la aorta ascendente son los más frecuentes $(50 \%)$, seguidos por la aorta descendente $(40 \%)$ y el arco aórtico $(10 \%)^{7}$. Alrededor de un $25 \%$ de los pacientes que presentan grandes AAT son portadores, además, de aneurismas abdominales ${ }^{1}$.

\section{Fisiopatología}

En la mayoría de los casos, los AAT ascendente son resultado de una necrosis quística de la media (NQM), que

\section{Figura 1.}

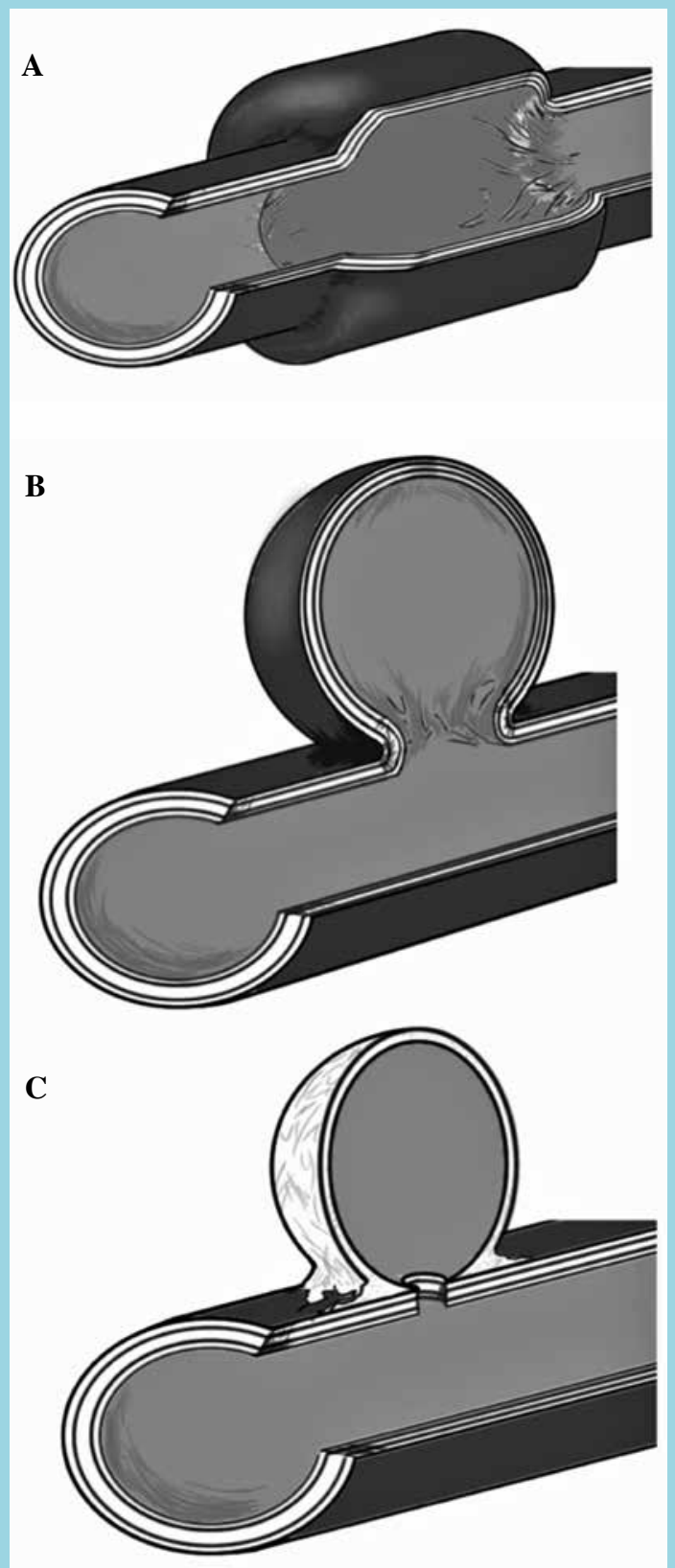

Figura 1.

a. Aneurisma fusiforme

b. Sacular

c.pseudoaneurisma

histológicamente corresponde a una pérdida de células musculares lisas y degeneración de fibras elásticas. Esto genera una debilidad de la pared arterial, que termina con la formación de un aneurisma. Si bien la NQM es un proceso que se desencadena con el paso de los años, la hi- 
pertensión arterial es un factor que lo acelera ${ }^{8}$. Teniendo en consideración el papel que desempeña la NQM, en el origen de los AAT, una serie de patologías ha sido relacionada con ambas entidades. Entre ellas destaca el Síndrome de Marfán, la válvula aórtica bicúspide y el Síndrome Familiar de Aneurisma de la Aorta Torácica.

El Síndrome de Marfán, se caracteriza por una mutación en uno de los genes que codifica la fibrilina-1, proteína estructural y componente esencial de las microfibrillas de elastina. Esto provoca una disminución, tanto en cantidad, como en organización de éstas.

Para demostrar las alteraciones histológicas presentes en pacientes portadores de válvula aórtica bicúspide, Fedak y cols. ${ }^{9}$ en el año 2003 , compararon a éstos con portadores de válvula aórtica tricúspide, llegando a la conclusión de que los primeros poseían una cantidad significativamente menor de fibrilina-1 en comparación con los segundos.

En la actualidad, la mayoría de los AAT esporádicos, que se presentan en ausencia de alteraciones del tejido conectivo, suelen tener un componente familiar. De ahí que algunos autores los agrupen dentro del Síndrome Familiar de Aneurisma de la Aorta Torácica. Un ejemplo de esto, se ve en el estudio Coady y cols. ${ }^{10}$ que, en el año 1999, demostró que al menos $19 \%$ de los pacientes tenían una historia familiar de AAT.

En lo que respecta a los AAT descendente, los cambios ateroscleróticos asociados a factores de riesgo cardiovascular desempeñan un rol primordial en la génesis de esta patología 1 . (Tabla 2)

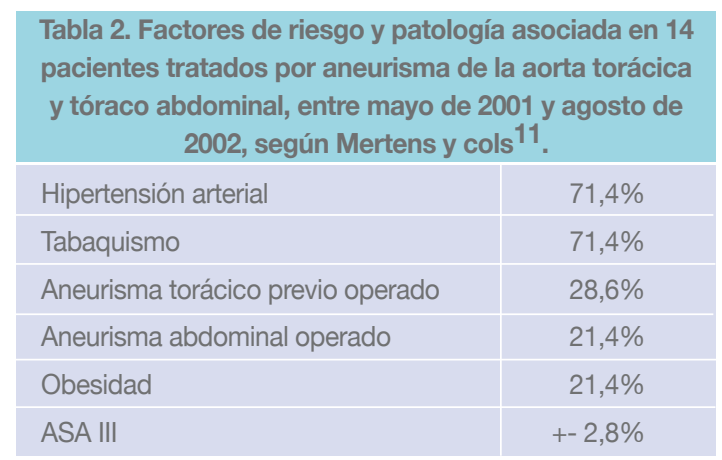

La disección aórtica crónica, también puede generar AAT ascendente y descendente. Al haber una debilidad de la pared arterial (que origina la disección) y una fragilidad de la pared más externa del lumen falso (que no cuenta con una pared intimal), se presenta una condición propicia para la formación aneurismática ${ }^{8}$.

Las infecciones (ej. endocarditis bacteriana, sífilis) y los procesos autoinmunes (ej. arteritis de Takayasu, espondi- litis anquilosante) también pueden originar aneurismas, ya sea por destrucción del colágeno y la fibras elásticas ${ }^{8}$, o bien por inflamación del fibrocartílago ${ }^{12}$, respectivamente.

\section{Manifestaciones Clínicas}

Los AAT suelen pesquisarse de manera incidental mediante exámenes imagenológicos solicitados por diferentes motivos de consulta ${ }^{13-14}$.

En el caso extraordinario de presentarse síntomas, estos van a depender de la región aórtica comprometida y la afectación de las estructuras adyacentes a ésta. Cuando la región afectada es la raíz aórtica o la aorta ascendente, el paciente puede presentar un soplo de insuficiencia aórtica o signos de insuficiencia cardíaca ${ }^{1}$.

Hay casos en que el aneurisma por efecto de masa genera una compresión directa de las estructuras intratorácicas, provocando diversos síntomas. Uno de estos es el dolor, que dependiendo de la localización, puede manifestarse a nivel cervical, torácico o abdominal. El dolor torácico suele referirse como una sensación de opresión retroesternal, irradiado a la región interescapular. El dolor abdominal por su parte, tiende a ubicarse en ambos flancos y a nivel de epigastrio ${ }^{2}$.

Cuando la vía aérea es la afectada, el paciente puede presentar tos, disnea, sibilancias, neumonía recurrente, hemoptisis o atelectasias. Si hay compresión del esófago predomina la disfagia y si hay compresión del nervio laringo recurrente habrá ronquido acompañado o no de pulsaciones de la laringe.

Se describe también el Síndrome de Robo Arterial (por afección de la arteria subclavia) y el Síndrome de la Vena Cava Superior. También pueden generarse trombos en el saco aneurismal, lo que derivará en complicaciones embólicas a distancia, ya sea en vasos coronarios, cerebrales, mesentéricos y/o de extremidades ${ }^{15}$.

A pesar de todo lo anteriormente expuesto lo más frecuente es que si el paciente debuta con síntomas, estos suelen deberse a una complicación como el Síndrome Aórtico Agudo o la rotura aneurismática, con el consiguiente dolor intenso de inicio brusco, en cuello, tórax y/o abdomen asociado a un importante compromiso hemodinámico ${ }^{1-16}$.

\section{Historia Natural}

La historia natural de los AAT no está completamente clarificada. Esto debido principalmente a que tanto la etiología como la ubicación influyen en el crecimiento de estos ${ }^{8}$. En términos generales los AAT tienen un crecimiento promedio de $0,1 \mathrm{~cm} / \mathrm{año}^{17}$. Diversos estudios demuestran que después de que un aneurisma alcanza un diámetro mayor a 
$6 \mathrm{~cm}$ en la aorta ascendente y mayor a $7 \mathrm{~cm}$ en la aorta descendente, el porcentaje de complicaciones aumenta considerablemente ${ }^{18}$. En un estudio Clouse et al. ${ }^{19}$ el riesgo de ruptura a 5 años de un AAT menor de $4 \mathrm{~cm}$ fue de $0 \%$; entre 4 y $5,9 \mathrm{~cm}$ de $16 \%$ y mayor o igual a $6 \mathrm{~cm}$ de $31 \%$. Mientras más rápido es el crecimiento del aneurisma, mayor es el riesgo de presentar complicaciones ${ }^{14}$. Dentro de los factores que aumentan el tamaño aneurismático destaca la edad avanzada, el género femenino, la enfermedad pulmonar obstructiva crónica, la hipertensión arterial, poseer una historia familar de AAT y la disección aórtica ${ }^{20}$. El pronóstico de un AAT roto es bastante ominoso. Johansson et al ${ }^{21}$, demostraron que solo el $41 \%$ de los pacientes que sufren una rotura aneurisática, llegan vivos al hospital.

\section{Diagnóstico}

A pesar de que existen AAT que no producen ningún tipo de anormalidad en una radiografía de tórax, hay ocasiones en que estos pueden ser visualizados. Los signos que apoyan su presencia son el ensanchamiento del mediastino, alargamiento del botón aórtico y la desviación de la tráquea $^{8}$. Debido a la inespecificidad de los hallazgos, se sugiere siempre complementar con otro estudio imagenológico.

La angiografía por tomografía computada (AngioTC) o por resonancia magnética (AngioRM), se perfilan como los exámenes de elección, ya que permiten determinar con exactitud la anatomía de la aorta y sus ramas, además del tamaño de aneurisma ${ }^{8-22-23}$ (Figura 2). La AngioTC permite una rápida adquisición de imágenes y la reconstrucción en 3D de la aorta ${ }^{24}$. La AngioRM también puede generar reconstrucciones en 3D y no utiliza radiación ionizante ni contraste yodado. Lamentablemente, requiere de a lo menos 30 minutos para ser efectuada y la cooperación del paciente para mantenerse en apnea ${ }^{24}$.

La ecocardiografía transtorácica (considerada anteriormente como el Gold Standard ${ }^{25}$ ), es un método no invasivo y altamente sensible para caracterizar el arco aórtico. Sin embargo, pierde sensibilidad cuando las alteraciones se presentan a nivel de la aorta ascendente y torácica. La ecocardiografía transesofágica, al ser un método invasivo, se reserva para la evaluación de los pacientes inestables o con sospecha de disección aórtica. Permite la visualización de toda la aorta torácica ${ }^{26}$.

La angiografía por sustracción digital es útil para la obtención de imágenes intraoperatorias durante el tratamiento endovascular de los AAT, ya que permite objetivar el lugar adecuado para orientar y desplegar la endoprótesis ${ }^{11}$.No suele utilizarse de rutina para el diagnóstico de esta patolo-
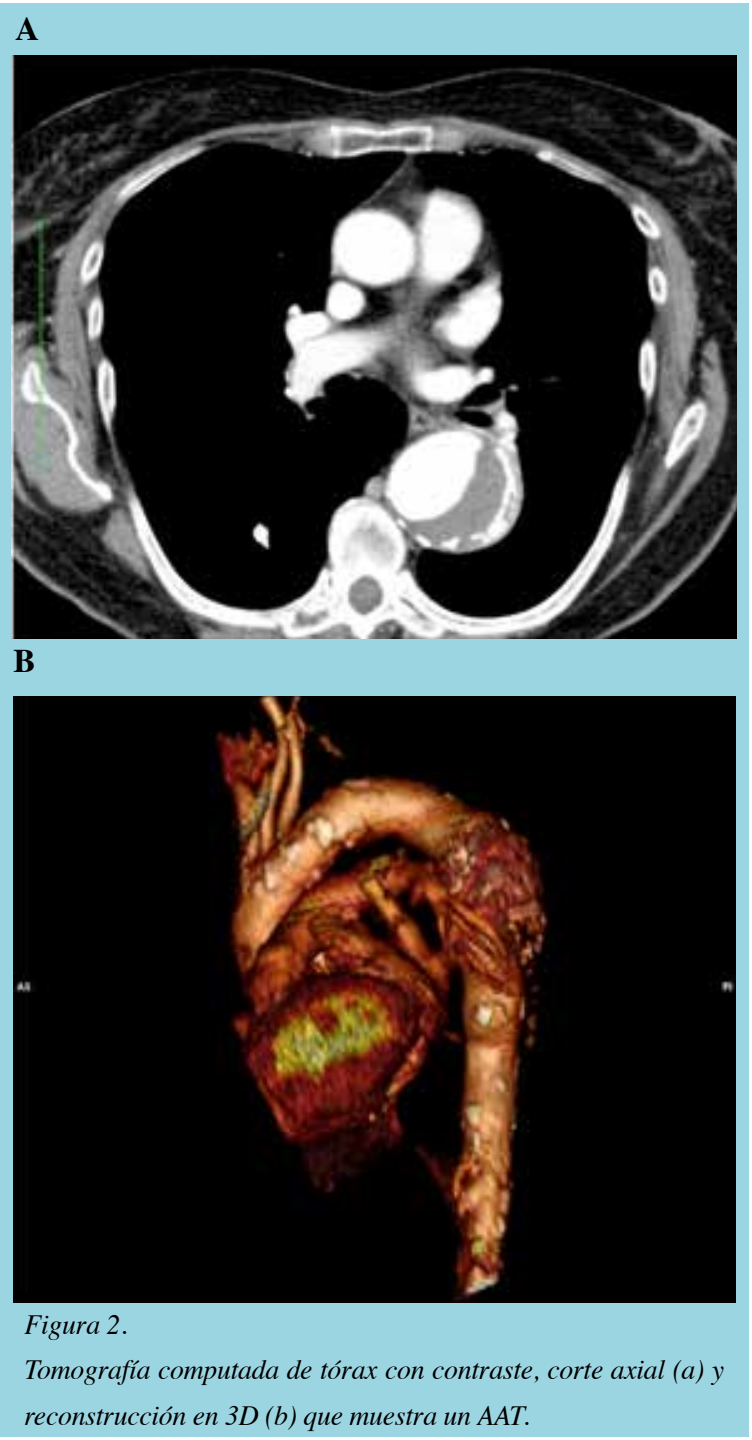

gía, debido a que visualiza únicamente la luz verdadera y no el diámetro total de la aorta; además, conlleva el riesgo de toxicidad por el medio de contraste, embolia y lesión arterial causada por el catéter o la guía utilizada.

\section{Tratamiento}

Además de las características del AAT, cada caso debe ser valorado de manera individual considerando factores como la edad, superficie corporal y patologías asociadas. Un control estricto de la presión arterial, la optimización del perfil lipídico y la cesación del hábito tabáquico, asociado a medidas orientadas a la reducción del riesgo de ateroesclerosis, son el pilar del tratamiento para los pacientes con aneurismas pequeños que no requieren cirugía ${ }^{2}$.

El manejo inicial está orientado a disminuir el stress sobre la pared, controlando la frecuencia cardíaca y la presión 
arterial, para así disminuir la probabilidad de que se produzca un aumento del tamaño del aneurisma o se complique con una ruptura. Dentro de los fármacos utilizados para esto, la primera opción son los beta bloqueadores con el objetivo de lograr una frecuencia cardíaca $\leq 60$ por minuto. En caso de estar contraindicado, los bloqueadores de canales de calcio son una alternativa. Si la presión arterial sistólica se mantiene sobre $120 \mathrm{mmHg}$ luego de controlada la frecuencia cardíaca, debe agregarse un inhibidor de la enzima convertidora de angiotensina $\mathrm{y} / \mathrm{u}$ otro vasodilatador $^{2}$.

Tabla 3. Indicaciones de tratamiento quirúrgico en aneuris-
mas de la aorta torácica ${ }^{1,2}$.
Diámetro > 50-55 mm en aorta ascendente, 55 mm en arco
aórtico, $60-70$ mm en aorta descendente y $45 \mathrm{~mm}$ en pacien-
tes con antecedentes de cirugía valvular aórtica
Presencia de síntomas
Crecimiento > 5 mm en 6 meses o 10 mm en 12 meses
Disección aórtica aguda tipo A
Insuficiencia aórtica severa

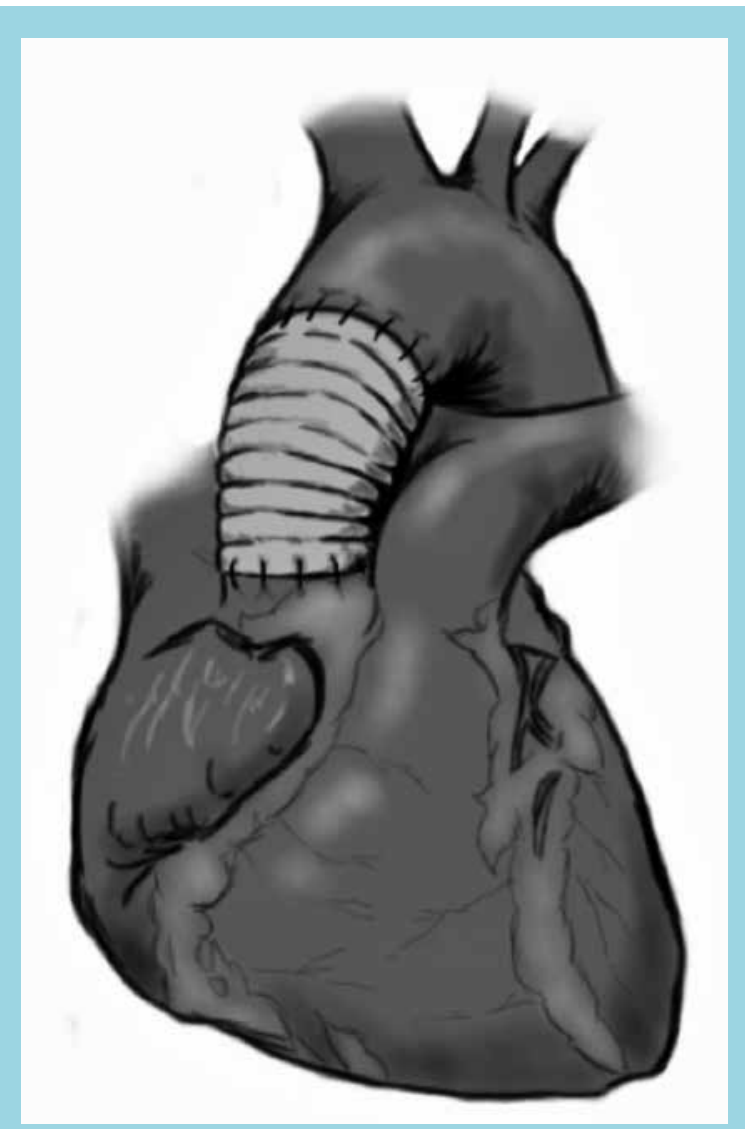

Figura 3.

Reemplazo de aorta ascendente con prótesis de Dacrón.

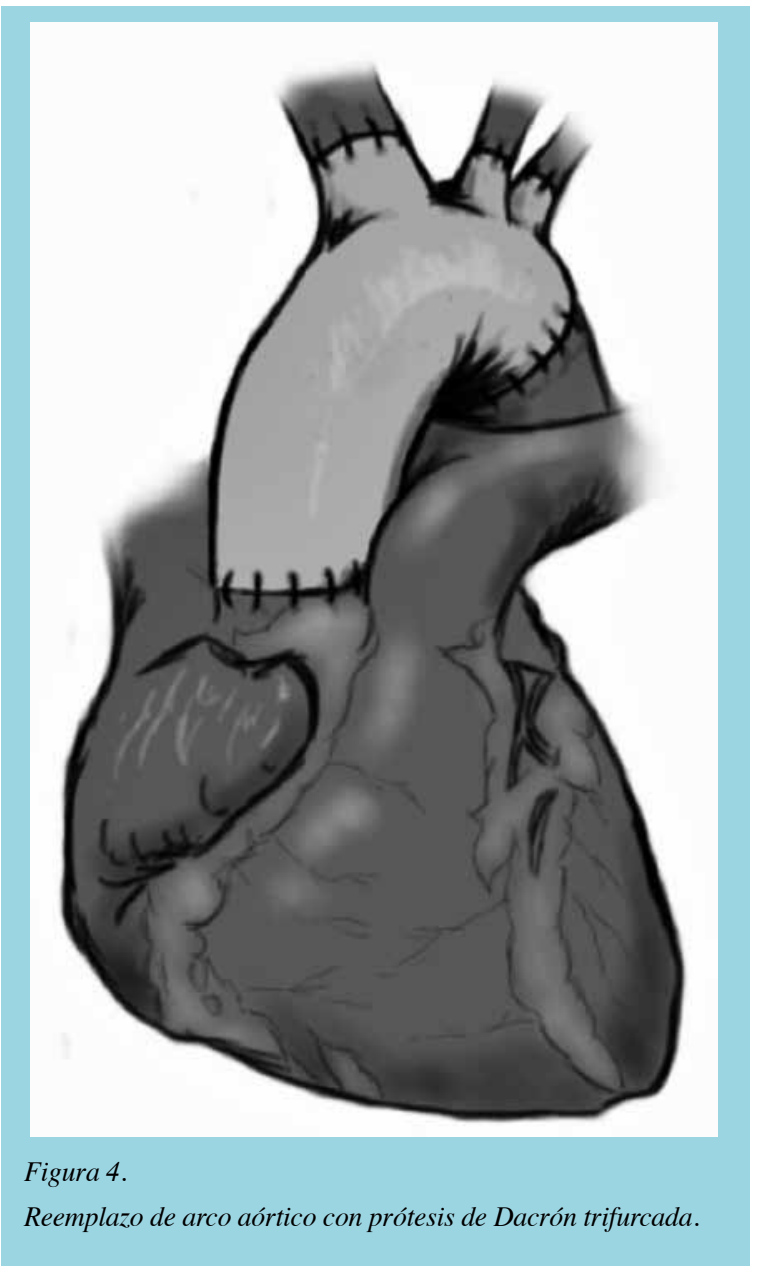

Las indicaciones de tratamiento quirúrgico se resumen en la Tabla 3. Para los pacientes con aneurisma de la aorta ascendente, el reemplazo aórtico por una prótesis de Dacrón es suficiente cuando no existe compromiso valvular (Técnica de David) ${ }^{27-28}$ (Figura 3). Al contrario, en el caso de los pacientes con Síndrome de Marfán, Loeys-Dietz, Ehlers-Danlos o cuando la válvula aórtica está dilatada, se realiza una sustitución de ésta y toda la aorta aneurismática por una prótesis valvulada con reimplantación de las arterias coronarias (Procedimiento de Bentall) ${ }^{2-29}$.

Para los aneurismas del cayado aórtico las opciones terapéuticas son tres; la cirugía abierta, el tratamiento endovascular y la combinación de ambas. En la primera se reseca el aneurisma incluyendo el origen de los grandes vasos (tronco braquiocefálico, arteria carótida común izquierda y arteria subclavia izquierda) y se reemplaza por una prótesis de Dacrón trifurcada que se anastomosa con cada una de las ramas, bajo apoyo de circulación extracorpórea ${ }^{7-30}$ (Figura 4). En la segunda, mediante un catéter se accede a la aorta torácica a través de la arteria femoral, donde se 


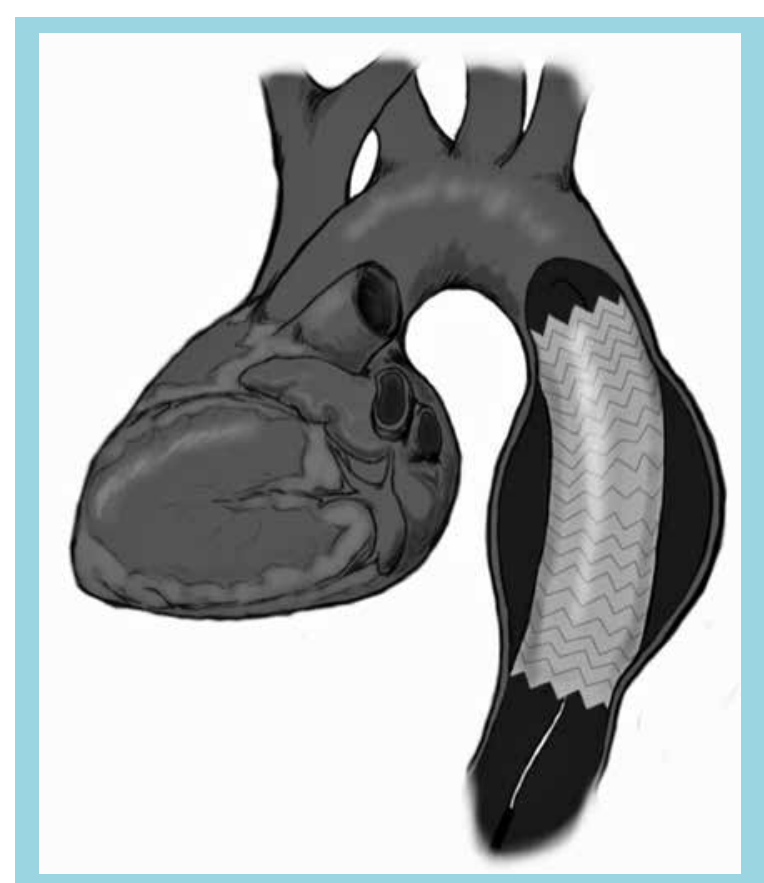

Figura 5.

Prótesis endovascular desplegada a nivel de aorta descendente.

libera e implanta una endoprótesis en el lumen aneurismático impidiendo que la sangre llegue al aneurisma ${ }^{31}$. En el tratamiento combinado, mediante una esternotomía media se realiza una derivación de los troncos supraaórticos o un "bypass" cervical extraanatómico con transposición de vasos, a lo que posteriormente se agrega la instalación de una prótesis endovascular. Lamentablemente, la desramificación abierta del cayado aumenta la complejidad y morbimortalidad de la intervención, anulando los efectos benéficos de la reparación endovascular. Esto ha llevado a que algunos centros utilicen prótesis ramificadas, fabricadas a medida ${ }^{32}$.

En el caso de los aneurismas de la aorta descendente se recomienda el tratamiento endovascular ${ }^{2}$ (Figura 5). Éste se realiza bajo anestesia general, para un mejor control de la hemodinamia y una inducción de hipotensión contro- $\operatorname{lada}^{28}$.

Las ventajas sobre la cirugía abierta son la anulación de grandes incisiones torácicas, menor pérdida de sangre, menor riesgo de isquemia medular (se evita el campleo aórtico y la ligadura de las arterias intercostales), menor dependencia de asistencia respiratoria mecánica y una recuperación más rápida ${ }^{33-35}$. Esto genera una disminución en la morbimortalidad operatoria.

\section{Seguimiento}

Los pacientes sin indicación de tratamiento quirúrgico o endovascular, deben ser controlados de manera seriada con estudios imagenológicos (AngioTC o AngioRM), con el fin de evaluar el crecimiento del aneurisma. Si además del aneurisma hay patología valvular asociada, se recomienda apoyar el estudio con ecocardiografía ${ }^{14}$.

Una conducta adecuada es volver a realizar el examen a los 6 meses de hecho el diagnóstico. Si el diámetro aórtico no ha variado en ese lapso de tiempo, el paciente puede controlarse cada 12 meses. En caso contrario el control sugerido es cada 3 o 6 meses $^{2}$. Si el aneurisma presenta un crecimiento acelerado ( $>0,5 \mathrm{~cm} / \mathrm{año})$, se recomienda la intervención ${ }^{14}$.

\section{Conclusión}

Considerando que en las últimas décadas ha habido un aumento en la incidencia y aún no hay una compresión completa de la fisiopatología involucrada, es necesario el desarrollo de estudios más acabados que permitan predecir de manera certera la evolución natural de esta patología.

A pesar de que se cuenta con una gran variedad de alternativas terapéuticas, el tratamiento ideal, que permita disminuir el tamaño aneurismático, no existe. Si bien el tratamiento endovascular está lejos de la perfección, hoy en día se ha consagrado como uno de los más efectivos en disminuir la morbimortalidad cuando se compara con el tratamiento quirúrgico. Es de esperar que surjan opciones menos invasivas que las actuales y así poder ofrecer a los pacientes de alto riesgo alternativas terapéuticas sin grandes riesgos asociados. 


\section{Referencias:}

1) FLEIG A, SEITZ K. Thoracic aortic aneurysms. Ultraschall Med. 2010; 31: 122-40.

2) HIRATZKA L, BAKRIS G, BECKMAN J, BERSIN R, CARR V, CASEY D, et al. 2010 ACCF/AHA/AATS/ACR/ASA/SCA/ SCAI/SIR/STS/SVM guidelines for the diagnosis and management of patients with Thoracic Aortic Disease: a report of the American College of Cardiology Foundation/American Heart Association Task Force on Practice Guidelines, American Association for Thoracic Surgery, American College of Radiology, American Stroke Association, Society of Cardiovascular Anesthesiologists, Society for Cardiovascular Angiography and Interventions, Society of Interventional Radiology, Society of Thoracic Surgeons, and Society for Vascular Medicine. Circulation. 2010; 121: e266-369.

3) HERRERA M, ALCÁNTARA M, LOMELÍ C, MENDOZA C, AGUIRRE C, LORENZO J, et al. Penetrating atherosclerotic ulcer of the thoracic aorta: endovascular treatment, hybrid or surgery. A review. Arch Cardiol Mex. 2009; 79: 140-6.

4) ÁlVAREZ H, BRAVO M, ARANCIBIA N, CHACÓN G. Pseudoaneurisma de la arteria temporal: Reporte de un caso y revisión de la literatura. Rev Chil Cir. 2006; 5: 469-472.

5) CLOUSE W, HALLETT J, SCHAFF H, GAYARI M, ILSTRUP D, MELTON L. Improved prognosis of thoracic aortic aneurysms: a population-based study. JAMA. 1998; 280: 1926-9.

6) GILLUM R. Epidemiology of aortic aneurysm in the United States. J Clin Epidemiol 1995; 48: 1289-1298.

7) KUZMIK G, SANG A, ELEFTERIADES J. Natural history of thoracic aortic aneurysms. J Vasc Surg. 2012; 56: 565-71.

8) ISSELBACHER E. Thoracic and abdominal aortic aneurysms. Circulation. 2005; 111: 816-828.

9) FEDAK P, DE SA M, VERMA S, NILI N, KAZEMIAN P, BUTANY J, et al. Vascular matrix remodeling in patients with bicuspid aortic valve malformations: implications for aortic dilatation. J Thorac Cardiovasc Surg. 2003; 126: 797-806.

10) COADY M, DAVIES R, ROBERTS M, GOLDSTEIN L, ROGALSKI M, RIZZO J, et al. Familial patterns of thoracic aortic aneurysms. Arch Surg. 1999; 134: 361-367.

11) MERTENS R, VALDES F, KRÄMER A, MARINE L, IRARRAZAVAL M, MORAN S, et al. Endovascular treatment of descending thoracic aorta aneurysm. Rev Med Chil. 2003; 131: $617-22$.

12) SIMKIN P. Acetabular osteitis in ankylosing spondylitis: Does fibrillin figure in its pathogenesis? J Rheumatol. 2001; 28: 2663-2666.

13) PRESSLER V, MCNAMARA J. Aneurysm of the thoracic aorta. Review of 260 cases. J Thorac Cardiovasc Surg. 1985; 89: 50 .

14) BOOHER A, EAGLE K. Diagnosis and management issues in thoracic aortic aneurysm. Am Heart J. 2011; 162: 38-46.

15) KOUCHOUKOS N, DOUGENIS D. Surgery of the thoracic aorta. New Eng J Med. 1997; 336: 1876-88.

16) HAHN S, KOTLER M, GOLDMAN A, ABLAZA S. Superior vena caval obstruction secondary to acute dissecting aneurysm of the aorta. Am Heart J. 1986; 111: 606-8.

17) DAVIES R, GOLDSTEIN L, COADY M, TITTLE S, RIZZO $\mathrm{J}$, KOPF G, et al. Yearly rupture or dissection rates for thoracic aortic aneurysms:simple prediction based on size. Ann Thorac Surg. 2002; 73: 17-28.

18) COADY M, RIZZO J, HAMMOND, MANDAPATI D, DARR $\mathrm{U}, \mathrm{KOPF} \mathrm{G}$, et al. What is the appropriate size criterion for resection of thoracic aortic aneurysms?. J Thorac Cardiovasc Surg. 1997; 113: 476-91.

19) CLOUSE W, HALLETT J, SCHAFF H, GAYARI M, ILSTRUP D, MELTON L. Improved prognosis of thoracic aortic aneurysmas: A population based study. JAMA. 1998; 280: 1926-1929.

20) SILVA J, MOTA P, SANTOS P, ANTUNES M, LEITÃO-MÁRQUEZ A. História natural de aneurisma de crossa da aorta: a necessidade de novas terapêuticas. Rev Port Cardiol. 2009; 28: 741-748.

21) JOHANSSON G, MARKSTROM U, SWEDENBORG J. Ruptured thoracic aortic aneurysms: a study of incidence and mortality rates. J Vasc Surg. 1995; 21: 985-8.

22) RUBIN G. Helical CT angiography of the thoracic aorta. J Thorac Imaging. 1997; 12: 128-49.

23) ROBERTS D. Magnetic resonance imaging of thoracic aortic 
aneurysm and dissection. Semin Roentgenol. 2001; 36: 295308.

24) BOOHER M, EAGLE K. Diagnosis and management issues in thoracic aortic aneurysm. Am Heart J. 2011; 162: 38-46.

25) CHEITLIN M, ARMSTRONG W, AURIGEMMA G, BELLER G, BIERMAN F, DAVIS J, et al. ACC/AHA/ASE 2003 guideline update for the clinical application of echocardiography: summary article: a report of the American College of Cardiology/American Heart Association Task Force on Practice Guidelines (ACC/AHA/ASE Committee to Update the 1997 Guidelines for the Clinical Application of Echocardiography). Circulation. 2003; 108: 1146-62.

26) BAILEY S, WEINER R, ALAGONA P, ANDERSON J, DECARA J, DOLOR R, et al. ACCF/ASE/AHA/ASNC/HFSA/ HRS/SCAI/SCCM/SCCT/SCMR 2011 Appropriate Use Criteria for Echocardiography. A Report of the American College of Cardiology Foundation Appropriate Use Criteria Task Force, American Society of Echocardiography, American Heart Association, American Society of Nuclear Cardiology, Heart Failure Society of America, Heart Rhythm Society, Society for Cardiovascular Angiography and Interventions, Society of Critical Care Medicine, Society of Cardiovascular Computed Tomography, Society for Cardiovascular Magnetic Resonance American College of Chest Physicians. J Am Soc Echocardiogr. 2011; 24: 229-67.

27) MARTÍNEZ H. Los aneurismas de la aorta torácica y su enfoque terapéutico. Arch. Cardiol. Méx 2006; 76: 124-136.

28) ALVES C, PALMA J, SOUZA J, CARVALHO A, BUFFOLO E. Endovascular treatment of thoracic disease: patient selec- tion and a proposal of a risk score. Ann Thorac Surg 2002; 73 : 1143-48.

29) BENTALL H, DE BONO A. A technique for complete replacement of the ascending aorta. Thorax 1968; 23: 338-339.

30) COSELLI J, CONKLIN L, LEMAIRE S. Thoracoabdominal aortic aneurysm repair: review and update of current strategies. Ann Thorac Surg 2002; 74: S1881-4.

31) PALMA J, CARVALHO A, BUFFOLO E, ALMEIDA D, GOMES W, BRASIL L: Endoscopic placement of stents in aneurysms of the descending thoracic aorta. Ann Thorac Surg 1998; 66: 256-258.

32) CRIADO F, BARNATAN M, RIZK Y, CLARK N, WANG C. Technical Strategies to Expand Stent-Graft Applicability in the Aortic Arch and Proximal Descending Thoracic Aorta. Journal of Endovascular Therapy 2002; 9: 32-38.

33) INOUE K, HOSOKAWA H, IWASE T, SATO M, YOSHIDA Y, UENO K, et al. Aortic arch reconstruction by transluminally placed endovascular branched stent graft. Circulation 1999; 100: II316-21.

34) TEMUDOM T, D'AYALA M, MARIN M, HOLLIER L, PARSONS R, TEODORESCU V, et al. Endovascular grafts in the treatment of thoracic aortic aneurysms and pseudoaneurysms. Ann Vasc Surg 2000; 14: 230-8.

35) Greenberg R, Resch T, Nyman U. Endovascular repair of descending thoracic aortic aneurysms: an early experience with intermediate term follow up. J Vasc Surg 2000; 31: 147-56. 